\title{
Antimicrobial use and resistance in Australia
}

\section{John Turnidge \\ Senior consultant Australian Commission on Safety and Quality in Health Care \\ Sydney}

\section{Keywords}

antibiotics, antibiotic resistance, bacterial infections

Aust Prescr 2017;40:2-3 http://dx.doi.org/10.18773/ austprescr.2017.007
Managing the emergence and increasing resistance to antimicrobials in hospitals and the community has become an urgent national and international problem. ' As part of a plan to tackle this, Australia is developing a coordinated national program to monitor antimicrobial use and resistance. ${ }^{2}$

In 2013, the Department of Health and the Department of Agriculture began to develop a 'one health' approach to resistance management, and released the National Antimicrobial Resistance Strategy in June $2015 .{ }^{3}$ One of the seven objectives was surveillance. The Australian Commission on Safety and Quality in Health Care was assigned the task of establishing this surveillance program, and set up the Antimicrobial Use and Resistance in Australia (AURA) project. The first national AURA report was released in June $2016 .{ }^{4}$

The Commission used a structured approach to ensure that all relevant data in human health were included. Both passive and targeted surveillance strategies were used to capture data on antimicrobial use and resistance. The Commission identified existing programs that were national or could become national:

- the National Antimicrobial Usage Surveillance Program (NAUSP) was collecting and publishing data on hospital antimicrobial use

- the Pharmaceutical Benefits Scheme (PBS) was collecting data on antimicrobial prescriptions in the community

- the National Antimicrobial Prescribing Survey was collecting data on appropriate use and compliance with guidelines in hospitals

- the NPS Medicinelnsight program was collecting data on appropriate use in general practice

- the Australian Group on Antimicrobial Use and Resistance was collecting resistance and some outcome data on selected pathogens causing bacteraemia originating in hospitals and in the community

- Queensland Health had a data cube capturing all antimicrobial resistance data across Queensland public hospitals (OrgTRx)

- Sullivan Nicolaides Pathology had antibiogram data from community and aged-care settings across Queensland and northern New South Wales
- the National Neisseria Network was collecting and reporting on resistance data for Neisseria gonorrhoeae and $N$. meningitidis

- the National Notifiable Diseases Surveillance System was collecting data on Mycobacterium tuberculosis from all mycobacterial reference laboratories.

The Commission reviewed these programs for suitability and national coverage, and enhanced and expanded them where necessary. This was largely achieved by the time the first national report was prepared. The report was prepared along similar lines to those generated by the benchmark countries in Scandinavia and the Netherlands, but also included data on appropriate antimicrobial use. The benchmark countries do not currently survey this.

The first AURA report focuses primarily on data from 2014, as this is the first year where complete data were available from all programs. ${ }^{4}$ Historical data were included when they were reliable and useful for interpretation. Where possible, comparisons with other countries were made on overall antibiotic use and on key pathogens.

The main findings in antimicrobial resistance data were:

- Rates of resistance in Escherichia coli in 2014 were $40-52 \%$ for ampicillin or amoxicillin, 20-30\% for trimethoprim (slightly lower for the trimethoprim/sulfamethoxazole combination), $18-21 \%$ for amoxicillin/clavulanate, 4-16\% for norfloxacin, and 0-10\% for ceftriaxone. Results depended on the clinical setting - public hospitals and residential aged-care facilities were associated with the higher resistance rates. About $13 \%$ of strains were resistant to more than three drug classes.

- Rates of methicillin-resistant Staphylococcus aureus (MRSA) were 11-28\% depending on the clinical setting. The highest rates were observed in public hospitals and residential aged care. Community-associated clones accounted for more invasive infections (such as bloodstream infections) than hospital clones. The incidence of invasive MRSA infections and the proportions of community-associated MRSA clones varied significantly between states and territories. 
- The prevalence of reduced susceptibility to ceftriaxone and azithromycin in N. gonorrhoeae was very low but is increasing slowly.

- $\quad$. meningitidis remains susceptible to the two main antimicrobials used for primary treatment (benzylpenicillin and ceftriaxone).

- Resistance to ampicillin, ceftriaxone and ciprofloxacin in Salmonella species is low except for human-associated 'typhoidal' serotypes.

- The proportion of multidrug resistant M. tuberculosis is low (<3\%).

- In terms of healthcare-associated pathogens, rates of resistance to key antimicrobials are quite low in Acinetobacter species and Pseudomonas aeruginosa.

The main findings on antimicrobial use were:

- Antimicrobial use in Australian hospitals is moderately high (936 defined daily doses per 1000 occupied bed days) when compared to similar countries that have data. However, there is evidence of a downward trend since 2010.

- In the national hospital survey in $2014,38 \%$ of patients were receiving antimicrobials on any given day. Of these, $77 \%$ were considered appropriate and $76 \%$ were compliant with national or local guidelines.

- In the 2015 pilot survey of residential agedcare facilities, $11 \%$ of patients were receiving antimicrobials but only $4.5 \%$ had a suspected or confirmed infection.

- Antimicrobial use in the community was very high in 2014 when compared to similar countries (see Fig.).

- Thirty million prescriptions for systemic and topical antimicrobials were dispensed on the PBS and 1164 prescriptions for systemic antimicrobials per 1000 inhabitants. The proportion of narrowspectrum antimicrobials prescribed was low (approximately 5\%).

- In the NPS Medicinelnsight program, excessive prescribing was identified for acute undifferentiated upper respiratory infection, acute bronchitis, tonsillitis, sinusitis and otitis media.
The AURA report collates valuable national information for the first time on antimicrobial use and resistance. Major areas for improvement in antimicrobial use have been identified in hospitals, residential aged care and especially in the community. The report provides baseline and some trend data on the resistances that are triggered by this use. The AURA program will continue to develop and refine its approach to national surveillance, and become a major part of the national strategy to contain antimicrobial resistance. It will provide the necessary data for monitoring the effects of interventions to reduce inappropriate use through stewardship and regulation, as described in the National Antimicrobial Resistance Strategy. ${ }^{3}<$

Conflict of interest: none declared

\section{Fig. Community antimicrobial use in Australia and other similar countries}

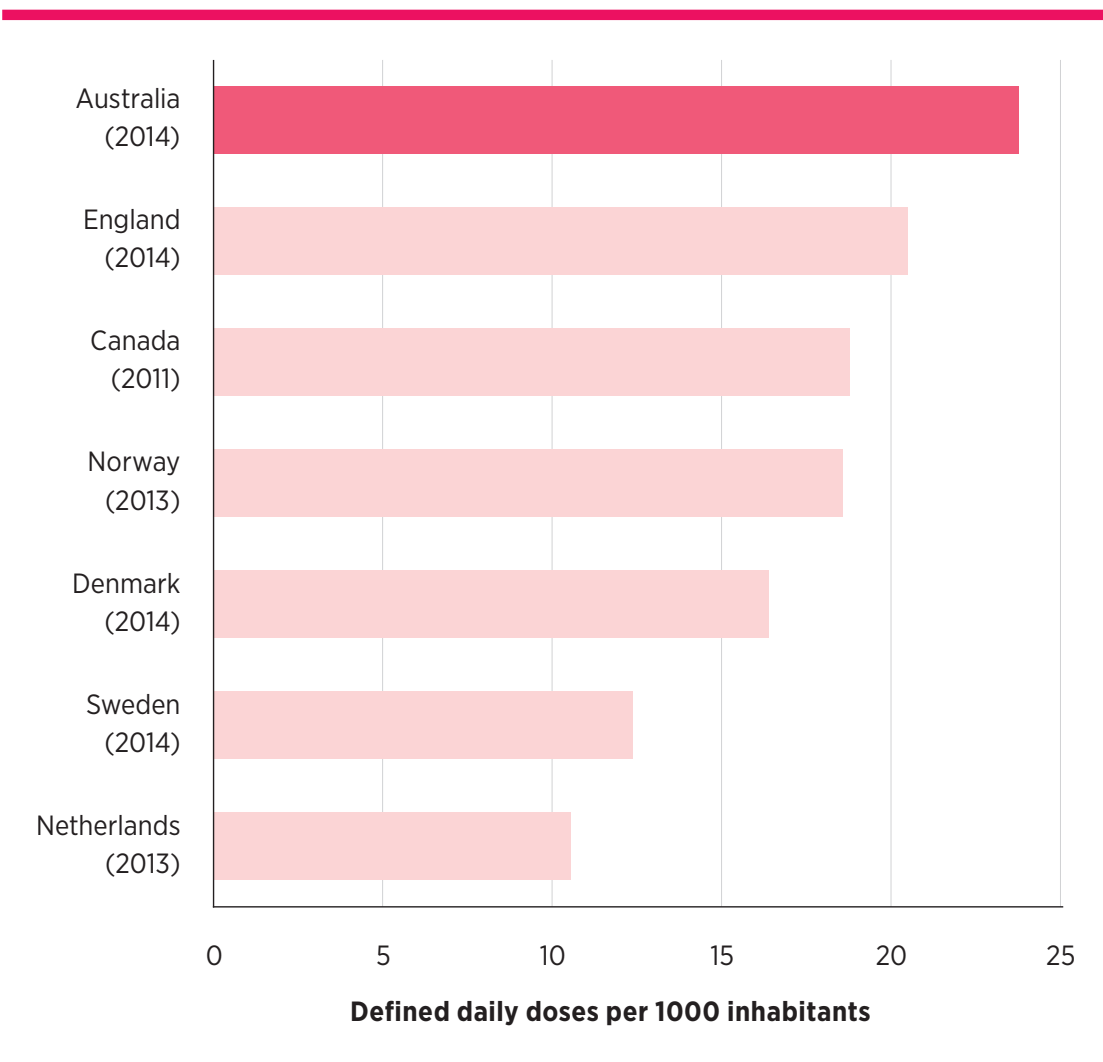

Source: Reference 4

\section{REFERENCES}

1. World Health Organization. Antimicrobial resistance: global report on surveillance. Geneva: WHO; 2014.

2. Joint Expert Technical Advisory Committee on Antibiotic Resistance. The use of antibiotics in food-producing animals: antibiotic-resistant bacteria in animals and humans. Canberra: Commonwealth of Australia; 1999.
3. Department of Health and Department of Agriculture. National Antimicrobial Resistance Strategy 2015-2019. Canberra: Commonwealth of Australia; 2015

4. Australian Commission on Safety and Quality in Health Care. AURA 2016: first Australian report on antimicrobial use and resistance in human health. Sydney: ACSQHC; 2016. 